\title{
SOME APPLICATIONS OF AN APPROXIMATION THEOREM FOR INVERSE LIMITS ${ }^{1}$
}

\section{MORTON BROWN}

Introduction. In $1954 \mathrm{C}$. E. Capel proved [1] the following theorems: Let $S$ be the inverse limit of a sequence of arcs (simple closed curves) where the bounding maps are onto and monotone. Then $S$ is an arc (simple closed curve). It may be noted that if $f$ is a monotone map of an arc (simple closed curve) onto itself, then $f$ is the uniform limit of a sequence of onto homeomorphisms. ${ }^{2}$ We call such a map a nearhomeomorphism. In this paper we prove the following two theorems: (1) If $S$ is the inverse limit of a sequence of copies of a given compact metric space $X$ and the bonding maps are near-homeomorphisms, then $S$ is homeomorphic to $X$. (2) Let $f: X \rightarrow Y, g: Y \rightarrow X$, where $f, g$ are maps and $X, Y$ are compact metric spaces. Suppose fg and gf are nearhomeomorphisms. Then $X$ is homeomorphic to $Y$. The second theorem follows directly from the first. In order to establish the first theorem we develop an approximation theorem which has interest in its own right.

Definitions and Notation. Let $X_{i}$ be a sequence of compact metric spaces, and for $i \geqq 2$ let $f_{i} \operatorname{map} X_{i}$ into $X_{i-1}$. Then the subspace ${ }^{3}$ $S=\left\{z \in \prod_{1}^{\infty} X_{i} \mid f_{i j}\left(z_{j}\right)=z_{i}\right\}$ of $\prod_{1}^{\infty} X_{i}$ is the limit space of the inverse system $\left(X_{i}, f_{i}\right)$; in notation $S=\operatorname{Lim}\left(X_{i}, f_{i}\right)$.

Let $f$ map $X$ into $Y$ where $X, Y$ are compact metric spaces. Then for $\epsilon>0: L(\epsilon, f)=\operatorname{Sup}\{\delta<d(X) \mid x, \quad y \in X$ and $|x-y|<\delta$ implies $|f(x)-f(y)|<\epsilon\} .{ }^{4}$ Since $X$ is compact, $0<L(\epsilon, f) \leqq d(X)$.

Let $\left(X_{i}, f_{i}\right)$ be an inverse system. A sequence $\left(a_{i}\right)$ of positive real numbers is a Lebesgue sequence for $\left(X_{i}, f_{i}\right)$ if there is a sequence $\left(b_{i}\right)$ of positive real numbers such that: (1) $\sum b_{i}<\infty$. (2) Whenever $x, y$ $\in X_{j}, i<j$, and $|x-y|<a_{j}$, then $\left|f_{i j}(x)-f_{i j}(y)\right|<b_{j}$. A sequence $\left(c_{i}\right)$ of positive real numbers is a measure for $\left(X_{i}, f_{i}\right)$ if: (1) $\sum_{n+1}^{\infty} c_{i}$ $<(1 / 2) c_{n}, n=1,2, \cdots,(2)$ for any two points $s, s^{\prime}$ of $\operatorname{Lim}\left(X_{i}, f_{i}\right)$ there is an integer $n$ such that $\left|s_{n+1}-s_{n+1}^{\prime}\right|>c_{n}$.

Presented to the Society April 18, 1959 under the title Inverse limits and Fréchet equivalence. Preliminary report; received by the editors September 1, 1959.

1 This paper was written while the author was an Office of Naval Research Fellow at the University of Michigan.

2 A simple proof for the case of an arc is the following: Suppose $f:[01] \rightarrow[01]$ is monotone. Obviously we may assume that $f(0)=0$, and $f(1)=1$. Let $h_{n}:[01] \rightarrow[01]$ by $h_{n}(x)=(1-1 / n) f(x)+(1 / n) x$.

${ }^{3} f_{i j}=f_{i+1} f_{i+2} \cdots f_{i}, f_{i i}=1$. If $z$ is a point of $X_{i}$ then $z_{i}$ will always denote the $i$ th coordinate of $z$. Hence $z=\left(z_{i}\right)$.

${ }^{4} d(X)$ denotes the diameter of $X \cdot|x-y|$ denotes the distance from $x$ to $y$. 
Lemma 1. Let $S=\operatorname{Lim}\left(X_{i}, f_{i}\right)$ where the $X_{i}$ are compact metric. Then $\left(X_{i}, f_{i}\right)$ has a Lebesgue sequence $\left(a_{i}\right)$.

Proof. Let $a_{j}=\operatorname{Min}_{i<j} L\left(2^{-i}, f_{i j}\right), b_{i}=2^{-i}$. Then $\sum b_{i}<\infty$, and whenever $x, y \in X_{j}, i<j$, and $|x-y|<a_{j}$, then $|x-y|<L\left(2^{-i}, f_{i j}\right)$. Hence $\left|f_{i j}(x)-f_{i j}(y)\right|<2^{-i}$.

Lemma 2. Let $S=\operatorname{Lim}\left(X_{i}, f_{i}\right)$ where the $X_{i}$ are compact metric. Then $\left(X_{i}, f_{i}\right)$ has a measure $\left(c_{i}\right)$.

Proof. Let $\left(a_{i}\right)$ be the Lebesgue sequence constructed in the proof of Lemma 1. Let $c_{1}=(1 / 2) a_{2}, c_{i+1}=(1 / 2) \operatorname{Min}\left[(1 / 2) c_{i}, a_{i+2}\right]$. Then $\left(c_{i}\right)$ is the required measure. For suppose $s, s^{\prime}$ are distinct points of $S$. Then there exist positive integers $n, j$ with $n<j$, and such that $\left|s_{n}-s_{n}^{\prime}\right|>2^{-i}$. If $\left|s_{j}-s_{j}^{\prime}\right| \leqq c_{j-1}$ then $\left|s_{j}-s_{j}^{\prime}\right|<a_{j}$. Hence $\left|s_{n}-s_{n}^{\prime}\right|$ $<2^{-i}$ which is absurd. Hence $\left|s_{j}-s_{j}^{\prime}\right|>c_{j-1}$.

Theorem 1. Let $S=\operatorname{Lim}\left(X_{i}, f_{i}\right), T=\operatorname{Lim}\left(X_{i}, g_{i}\right)$ where the $X_{i}$ are compact metric spaces. Suppose $\left\|f_{i+1}-g_{i+1}\right\|<a_{i}, i=1,2, \cdots$, where $\left(a_{i}\right)$ is a Lebesgue sequence for $\left(X_{i}, g_{i}\right)$. Then the function $F_{N}: S \rightarrow X_{N}$ defined by $F_{N}=\operatorname{Lim}_{n} g_{N n} f_{n}$ is well defined and continuous. ${ }^{5}$ Moreover, the function $F: S \rightarrow T$ defined by $F(s)=\left(F_{1}(s), F_{2}(s), \cdots,\right)$ is well defined, continuous, and onto.

Proof. Let $N$ be fixed. Let $\left(b_{i}\right)$ be the sequence associated with $\left(a_{i}\right)$.

$$
\operatorname{Lim}_{i \rightarrow \infty ; N<i<j}\left\|g_{N i} f_{i j}-g_{N i} g_{i j}\right\|=0 .
$$

PROOF OF (1.1). $\left\|g_{N i} f_{i j}-g_{N i} g_{i j}\right\|=\sum_{r=1}^{j-1}\left\|g_{N i} g_{i r} f_{r j}-g_{N i} g_{i r+1} f_{r+1}\right\|$. Now $g_{N i} g_{i r} f_{r j}=g_{N r} f_{r+1} f_{r+1} j$, and $g_{N i} g_{i r+1} f_{r+1 j}=g_{N r} g_{r+1} f_{r+1} j$. Since $\left\|g_{N r} f_{r+1} f_{r+1} j-g_{N r} g_{r+1} f_{r+1}\right\| \leqq\left\|g_{N r} f_{r+1}-g_{N r} g_{r+1}\right\|,\left\|g_{N i} f_{i j}-g_{N i} g_{i j}\right\|$ $\leqq \sum_{r=1}^{j-1}\left\|g_{N r} f_{r+1}-g_{N r} g_{r+1}\right\|$. But the hypothesis assures that $\left\|f_{r+1}-g_{r+1}\right\|$ $<a_{r .}$. Hence $\left\|g_{N_{r}} f_{r+1}-g_{N r} g_{r+1}\right\|<b_{r}$. Hence $\left\|g_{N i} f_{i j}-g_{N i} g_{i j}\right\| \leqq \sum_{r=i}^{j-1} b_{r}$ $\leqq \sum_{r=1}^{\infty} b_{r}$. Finally $\operatorname{Lim}_{i \rightarrow \infty}\left\|g_{N i} f_{i j}-g_{N i} g_{i j}\right\| \leqq \operatorname{Lim}_{i \rightarrow \infty} \sum_{r=i}^{\infty} b_{r}=0$.

$$
\operatorname{Lim}_{i \rightarrow \infty ; i<j}\left\|g_{N i} f_{i \infty}-g_{N j} f_{j \infty}\right\|=0 .
$$

The proof of (1.2) precisely parallels that of (1.1).

$$
\operatorname{Lim}_{i \rightarrow \infty} g_{N i} f_{i \infty}(s)=F_{N}(s) \text { uniformly in } s .
$$

(1.3) follows immediately from (1.2).

(1.4) Hence $F_{N}=\operatorname{Lim}_{i \rightarrow \infty} g_{N i} f_{i \infty}$ is well defined and continuous.

${ }^{5} f_{n_{\infty}}$ is the map projecting each point of $S$ onto its $n$th coordinate. 
From this it follows immediately that $F: S \rightarrow \prod_{i=1}^{\infty} X_{i}$ is well defined and continuous.

(1.5) $F(S) \subset T$. To prove (1.5) it suffices to show that

$$
\begin{aligned}
g_{i j} F_{j}(s) & =F_{i}(s) \\
g_{i j} F_{j}(s) & =g_{i j} \operatorname{Lim}_{n \rightarrow \infty} g_{j n} f_{n \infty}(s) \\
& =\operatorname{Lim}_{n \rightarrow \infty} g_{i j} g_{j n} f_{n \infty}(s) \\
& =\operatorname{Lim}_{n \rightarrow \infty} g_{i n} f_{n \infty}(s) \\
& =F_{i}(s) .
\end{aligned}
$$

(1.6) $F: S \rightarrow T$ is onto.

Proof of (1.6). Let $t=\left(t_{n}\right) \in T$. Let $N$ be fixed. We first show that there is an $s^{N} \in S$ such that $F_{N}\left(s^{N}\right)=t_{N}$. Let $\epsilon>0$. By (1.1), (1.3), and the convergence of $\sum_{i=1}^{\infty} b_{i}$, there is an $i>N$ such that $\left\|F_{N}-g_{N i} f_{i \infty}\right\|$ $<\epsilon / 3,\left\|g_{N i} f_{i j}-g_{N i} g_{i j}\right\|<\epsilon / 3$ all $j>i$; and $b_{i}<\epsilon / 3$. Fix this $i$. Now by Corollary 3.8, Chapter VIII of [2] $\bigcap_{j=i}^{\infty} f_{i j}\left(X_{j}\right)=f_{i \infty}(S)$. Since the $X_{j}$ are compact there is a $j>i$ such that each point of $f_{i j}\left(X_{j}\right)$ is of distance less than $a_{i}$ from a point of $f_{i \infty}(S)$. Hence there is a point $s \in S$ such that $\left|f_{i j}\left(t_{j}\right)-f_{i \infty}(s)\right|<a_{i}$. Hence $\left|g_{N i} f_{i j}\left(t_{j}\right)-g_{N i} f_{i \infty}(s)\right|<b_{i}<\epsilon / 3$. Then

$$
\begin{aligned}
\left|F_{N}(s)-t_{N}\right| \leqq & \left|F_{N}(s)-g_{N i} f_{i \infty}(s)\right|+\left|g_{N i} f_{i \infty}(s)-g_{N i} f_{i j}\left(t_{j}\right)\right| \\
& +\left|g_{N i} f_{i j}\left(t_{j}\right)-g_{N i} g_{i j}\left(t_{j}\right)\right| \\
\leqq & \epsilon / 3+\epsilon / 3+\epsilon / 3=\epsilon .
\end{aligned}
$$

Hence the compactness of $S$ insures the existence of an $s^{N} \in S$ such that $F_{N}\left(s^{N}\right)=t_{N}$. Now for all $N, F_{N}\left(s^{N}\right)=t_{N}$ implies that $F_{i}\left(s^{N}\right)=t_{i}$ for $i<N$. If $s \in S$ is a convergence point of the set $\left\{s^{N}\right\}$ then $F(s)=t$.

REMARK. $F$ is not necessarily $1-1$. For suppose $T=\operatorname{Lim}\left(I_{i}, g_{i}\right)$ where $I_{i}$ is the unit interval [01] , and $g_{i}(t)=0, t \in I_{i}$. Let $S=\operatorname{Lim}\left(I_{i}, f_{i}\right)$ where $f_{i}(t)=t, t \in I_{i}$. Let $a_{i}=2$ for all $i$. Then $\left(a_{i}\right)$ is a Lebesgue sequence for $\left(I_{i}, g_{i}\right)$ where $b_{i}=2^{-i}$. For if $x, y \in I_{j}$ then $\left|g_{i j}(x)-g_{i j}(y)\right|$ $=0<2^{-i}$. Also $\left|f_{i+1}(t)-g_{i+1}(t)\right|$ is $t$, and $t<a_{i}$. Since $S$ is an arc and $T$ is a point, $F$ cannot be $1-1$.

Theorem 2. Let $S=\operatorname{Lim}\left(X_{i}, f_{i}\right), T=\operatorname{Lim}\left(X_{i}, g_{i}\right)$ where the $X_{i}$ are compact metric spaces. Suppose

$$
\left\|f_{i}-g_{i}\right\|<\min \left[c_{i-1} ; \min _{k<i-1} L\left(c_{i-1}, g_{k i-1}\right)\right]
$$


where $\left(c_{i}\right)$ is a measure for $\left(X_{i}, f_{i}\right)$. Then the map $F: S \rightarrow T$ described in Theorem 1 is a homeomorphism onto.

Proof. To prove that $F$ is well defined continuous and onto, it suffices to show that $a_{i}=\min _{k<i} L\left(c_{i}, g_{k i}\right)$ is a Lebesgue sequence for $\left(X_{i}, g_{i}\right)$. The sequence we associate with $\left(a_{i}\right)$ is $\left(c_{i}\right)$. Since $c_{N}$ $>2 \sum_{N+1}^{\infty} c_{i}, \quad \sum_{1}^{\infty} c_{i}<\infty$. Finally, suppose $x, y \in X_{j}, i<j$, and $|x-y|<a_{j}$. Then $|x-y|<L\left(c_{j}, g_{i j}\right)$. Hence $\left|g_{i j}(x)-g_{i j}(y)\right|<c_{j}$.

Proof of (2.1).

$$
\left\|f_{N i}-g_{N i}\right\|<\sum_{j=N}^{\infty} c_{j}, \quad \text { all } N, i,(N<i) .
$$

$$
\begin{aligned}
\left\|g_{N i}-f_{N i}\right\| & \leqq\left\|g_{N i-1} g_{i}-g_{N i-1} f_{i}\right\|+\left\|g_{N i-1} f_{i}-f_{N i-1} f_{i}\right\|, \\
& \leqq\left\|g_{N i-1} g_{i}-g_{N i-1} f_{i}\right\|+\left\|g_{N i-1}-f_{N i-1}\right\| .
\end{aligned}
$$

Since $\left\|g_{i}-f_{i}\right\|<a_{i-1}, \quad\left\|g_{N i-1} g_{i}-g_{N i-1} f_{i}\right\|<c_{i-1}$. Hence

$$
\left\|g_{N i}-f_{N i}\right\|<c_{i-1}+\left\|g_{N i-1}-f_{N i-1}\right\| \text {. }
$$

Continuing recursively, $\left\|g_{N i}-f_{N i}\right\|<c_{i-1}+\cdots+c_{N}$.

$$
F \text { is } 1-1 \text {. }
$$

Proof of (2.2). Suppose $F(s)=F\left(s^{\prime}\right), s, s^{\prime} \in S$. If $s \neq s^{\prime}$, then for some $n>1, c_{n-1}<\left|s_{n}-s_{n}^{\prime}\right|$. Now for $i>n$,

$$
\begin{aligned}
c_{n-1} & <\left|s_{n}-s_{n}^{\prime}\right|=\left|f_{n i}\left(s_{i}\right)-f_{n i}\left(s_{i}^{\prime}\right)\right| \\
& \leqq\left|f_{n i}\left(s_{i}\right)-g_{n i}\left(s_{i}\right)\right|+\left|g_{n i}\left(s_{i}\right)-g_{n i}\left(s_{i}^{\prime}\right)\right|+\left|g_{n i}\left(s_{i}^{\prime}\right)-f_{n i}\left(s_{i}^{\prime}\right)\right| .
\end{aligned}
$$

Applying (2.1) we get:

$$
c_{n-1}<2 \sum_{j=n}^{\infty} c_{j}+\left|g_{n i}\left(s_{i}\right)-g_{n i}\left(s_{i}^{\prime}\right)\right|, \quad \text { all } i>n .
$$

Letting $i$ approach infinity, $\left|g_{n i}\left(s_{i}\right)-g_{n i}\left(s_{i}^{\prime}\right)\right| \rightarrow\left|F_{n}(s)-F_{n}\left(s^{\prime}\right)\right|=0$. Hence (2.3) becomes $c_{n-1} \leqq 2 \sum_{j=n}^{\infty} c_{j}$. But this contradicts the requirement that $c_{n-1}>2 \sum_{j=n}^{\infty} c_{j}$.

Theorem 3. Let $S=\operatorname{Lim}\left(X_{i}, f_{i}\right)$ where the $X_{i}$ are compact metric spaces. For $i \geqq 2$ let $K_{i}$ be a nonempty collection of maps from $X_{i}$ into $X_{i-1}$. Suppose that for each $i \geqq 2$ and $\epsilon>0$ there is $g \in K_{i}$ such that $\left\|f_{i}-g\right\|<\epsilon$. Then there is a sequence $\left(g_{i}\right)$ where $g_{i} \in K_{i}$ and $S$ is homeomorphic to $\operatorname{Lim}\left(X_{i}, g_{i}\right)$.

Proof. Let $\left(c_{i}\right)$ be a measure for $\left(X_{i}, f_{i}\right)$. Let $g_{2}$ be any element of $K_{2}$. Let $g_{3}$ be an element of $K_{3}$ such that $\left\|f_{3}-g_{3}\right\|<L\left(c_{2}, g_{2}\right)$. Inductively, let $g_{n+1}$ be an element of $K_{n+1}$ such that $\left\|f_{n+1}-g_{n+1}\right\|$ 
$<\min _{k<n} L\left(c_{n}, g_{k n}\right)$. (Here $\left.g_{k n}=g_{k+1} g_{k+2} \cdots g_{n}\right)$. Then by Theorem 2 if $T=\operatorname{Lim}\left(X_{i}, g_{i}\right), F: S \rightarrow T$ homeomorphically onto.

Near homeomorphisms. Let $X$ be a metric space. A map $f: X \rightarrow X$ is a near homeomorphism if for any $\epsilon>0$ there is a homeomorphism $H_{\epsilon}$ of $X$ onto itself such that $\left\|H_{\epsilon}-f\right\|<\epsilon$.

THEOREM 4. Let $S=\operatorname{Lim}\left(X_{i}, f_{i}\right)$ where the $X_{i}$ are all homeomorphic to a compact metric space $X$, and for all $i, f_{i}$ is a near homeomorphism. Then $S$ is homeomorphic to $X$.

Proof. By Theorem 3 there is a sequence $h_{i}$ of homeomorphisms of $X_{i}$ onto $X_{i-1}$ such that $S=\operatorname{Lim}\left(X_{i}, h_{i}\right)$. But now $S$ must be homeomorphic to $X$.

Corollary. Let $S=\operatorname{Lim}\left(X_{i}, f_{i}\right)$ where each $X_{i}$ is a copy of a fixed 2-manifold $X$ (compact and with or without boundary) and $f_{i}$ is monotone onto. Then $S$ is homeomorphic to $X$.

Proof. J. W. T. Youngs [4] has proven that if $f: X \rightarrow X$ is monotone onto and $X$ is a compact 2 manifold (with or without boundary) then $f$ is a near homeomorphism.

Theorem 5. Let $X, Y$ be compact metric spaces. Suppose $f$ maps $X$ into $Y, g$ maps $Y$ into $X$, and $f g$ and $g f$ are near homeomorphisms. Then $X$ is homeomorphic to $Y$.

Proof. The following diagram commutes:

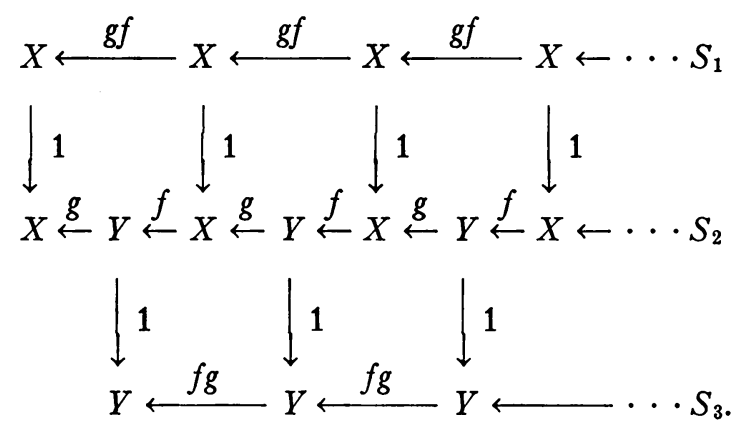

Hence $S_{1}, S_{2}$, and $S_{3}$ are mutually homeomorphic. But by Theorem 4 $S_{1}$ is homeomorphic to $X$ and $S_{3}$ is homeomorphic to $Y$.

Corollary. Let $X, Y$ be compact metric spaces. Suppose $f$ maps $X$ onto $Y, g$ maps $Y$ into $X, g f$ is a near homeomorphism. Then $\operatorname{Dim}(X)$ $\leqq \operatorname{Dim}(Y)$.

Proof. Examination of the diagram for Theorem 5 yields that $X$ can be expressed as the inverse limit of $Y$ 's. Hence $\operatorname{Dim}(X) \leqq \operatorname{Dim}(Y)$. 


\title{
REFERENCES
}

1. C. E. Capel, Inverse limit spaces, Duke Math. J. vol. 21 (1954) pp. 233-245.

2. S. Eilenberg and N. E. Steenrod, Foundations of algebraic topology, Princeton University Press, 1952.

3. Hans Freudenthal, Entwicklungen von Raumen und ihrer Gruppen, Compositio Math. vol. 4 (1937) pp. 145-234.

4. J. W. T. Youngs, Homeomorphic approximations to monotone mappings, Duke Math. J. vol. 15 (1948) pp. 87-94.

UNIVERSITY OF MICHIGAN

\section{NEW METHOD FOR EXPANSION AND CONTRACTION MAPS IN UNIFORM SPACES}

\author{
THOMAS A. BROWN ${ }^{1}$ AND W. W. COMFORT ${ }^{2}$
}

1. Introduction and definitions. In [2], Freudenthal and Hurewicz showed that if the function $f$, from the totally bounded metric space $M$ onto $M$, has the property that $(f x, f y) \leqq(x, y)$ for each $x$ and $y$ in $M$, then $f$ is an isometry. By amplifying the sequential argument given in [2], Rhodes (see [4]) proved that an even stronger result holds in the more general setting of uniform spaces. Using a different method, the present paper offers a theorem similar to that of Rhodes, together with a number of results concerning "expansion" maps in uniform spaces. The notation used here, which very closely approximates that of [4], has been taken from Chapter 6 of [3].

1.1. Definition. If $(M, \mathfrak{U})$ is a uniform space, then a subset $B$ of $u$ will be called a basis for $(M, \mathfrak{u})$ if

(a) if $x \in M$ and $U \in B$, then $(x, x) \in U$;

(b) if $U \in \mathcal{U}$, then $U^{-1}$ contains a member of $B$;

(c) for each $U \in \mathcal{U}$ there is a $V \in B$ for which $V \circ V \subset U$; and

(d) for each $U \in \mathcal{U}$ and $V \in \mathcal{U}$, there is a $W \in \mathbb{B}$ for which $W \subset U \cap V$.

1.2. Definition. If $B$ is a basis for the uniform space $(M, \mathfrak{u})$, then $B$ is said to be open if each of its elements is open in $M \times M$.

1.3. Definition. If $B$ is a basis for the uniform space $(M, \mathfrak{u})$, then $B$ is said to be ample if, whenever $(x, y) \in U \in B$, there is a $W \in ß$ for which $(x, y) \in W \subset \bar{W} \subset U$.

Received by the editors September 7, 1959.

${ }^{1}$ Research conducted while a pre-doctoral fellow of the National Science Foundation.

2 Research supported by Office of Ordnance Research, U. S. Army, under contract DA-19-020-ORD-3778. 